Калов Х.M. Калов P.X.*

\title{
АКТИВНЫЕ ВОЗДЕЙСТВИЯ НА ТЕПЛЫЕ ТУМАНЫ И ИНСТРУМЕНТАЛЬНОЕ ОПРЕДЕЛЕНИЕ ИХ ЭФФЕКТИВНОСТИ
}

Введение
Материалы и методы исследований
Несмотря на наличие большого количества теоретических и экспериментальных работ российских и зарубежных авторов (например, [1-3]) проблема рассеяния теплых туманов и низких слоистых облаков $(\mathrm{HCO})$ до настоящего времени остается ещё нерешенной до конца. Из всего многообразия методов рассеяния теплых туманов, предложенных к настоящему времени только тепловые наиболее близки к достижению ожидаемого результата Недостаточно полно решена задача инструментального определения горизонтальных и вертикальных протяженностей просветленной зоны в результате искусственного рассеяния туманов и НСО.

В работе разработан тепловой метод рассеяния теплых туманов и НСО основанный на использовании высокотемпературных источников тепповой энергии. В качестве таких источников использовались взрывы тепловых зарядов, при которых повышается температура и давление в тумане и НСО. Это повышение приводит к испарению тумана в зоне воздействия. Разработано лазерно-локационное устройство для оценки физической эфффективности искусственного рассеяния теплых туманов и НСО. Устройство используется в стационарных условиях на земле и на борту самолета-лаборатории Як-40.

Результаты исследований

и их обсуждение. При тепловом методе рассеяния туманов и НСО под действием повышения давления и температуры при взрыве в облачной среде капли, пересекающие фронт ударной волны, дробятся на более мелкие капельки и испаряются. Получены формулы для расчета: избыточного давления и температуры в тумане при взрыве тепловых зарядов; времени полного испарения капель и радиуса зоны просветления в тумане. Расчеты показали, что избыточное давление и температура в тумане при взрыве теплового заряда увеличиваются с увеличением величины заряда. Температура на фронте ударной волны ещё заметно выше температуры невозмущенного воздуха на расстояниях до 200 метров от места взрыва. Если взрыв теплового заряда происходит на небольшой высоте (1...2 м) над грунтом, то полное испарение капель тумана наблюдается в радиусе до 800 и более метров.

Выводы.

Разработанный нами тепловой метод дает возможность создавать зоны просветления небольшими взрывами теплового заряда под действием повышения температуры и давления на волне сжатия по сравнению с их значениями в невозмущенном воздухе. При взрыве небольшого заряда возникает облако расширяющихся взрывных газов, тепловое излучение которого испаряет капельки облачного образования и создает зону просветления в тумане до 800 м. Лазерно-локационное устройство с лазерным излучателем И3-25-1 на длине волны 0,532 мкм с высокой точностью (4...5 м) определяет границы зоны рассеяния тумана.

Ключевые слова: искусственное рассеяние туманов, результаты активных воздействий лазерно-локационное устройство, лазерный излучатель. 
Kalov Kh.M. Kalov R.Kh.

Introduction.

Materials and methods of research.

Results of the study and their Discussion

Conclusions

Key words:
High Mountain Geophysical Institute, Nalchik, Russia

\section{Active impacts on warm fogs and instrumental determination of their efficiency}

Despite the presence of a large number of theoretical and experimental works of Russian and foreign authors (for example [1-3]), the problem of scattering of warm fogs and low layered clouds (LLC) remains unresolved to the present time. Of the variety of warm fog scattering methods proposed to date, only thermal ones are the closest to achieving the expected result. Not fully solved the problem of the instrumental determination of the horizontal and vertica extents of the zone illuminated by artificial dissipation of fog and LLC.

In this paper, a thermal method of methods of scattering of warm fogs and LLC based on the use of high-temperature sources of thermal energy. The sources used were explosions of thermal charges, which increased the temperature and pressure in the fog and LLC. This rise leads to the evaporation of the mist in the treated area. Developed laser radar device to measure the physical efficiency of the artificial dissipation of warm fog and LLC. The device is used in stationary conditions on the ground and on Board the Yak-40 laboratory aircraft.

Under the thermal method of fog and LLC scattering, droplets crossing the shock wave front are crushed into smaller droplets and evaporate under the action of increasing pressure and temperature during the explosion in a cloud medium. The formulas for calculating the excess pressure and temperature in the fog during the explosion of thermal charges, the time of complete evaporation of droplets and the radius of the zone of enlightenment in the fog are obtained. Calculations have shown that the excess pressure and temperature in the fog in the explosion of heat charge increase with increasing charge The temperature at the front of the shock wave is still noticeably higher than the temperature of the undisturbed air at distances up to 200 meters from the explosion site. If the explosion of heat charge occurs at a low altitude $(1 \ldots 2$ $\mathrm{m}$ ) above the ground, the complete evaporation of fog drops is observed in a radius of $800 \mathrm{~m}$ and more.

The thermal method developed by us makes it possible to create zones of enlightenment by small explosions of a thermal charge under the influence of temperature and pressure increase on a compression wave in comparison with their values in the unperturbed air. When a small charge explodes, a cloud of expanding explosive gases appears, the thermal radiation of which evaporates droplets of cloud formation and creates a zone of enlightenment in the fog up to $800 \mathrm{~m}$. the Laser-locating device with a laser emitter IZ-25-1 at a wavelength of $0.532 \mu \mathrm{m}$ with high accuracy $(4 \ldots 5 \mathrm{~m})$ determines the boundaries of the fog scattering zone.

artificial dissipation of fogs, the results of active effects, laser ranging device laser emitter 


\section{Введение}

Проблема искусственного рассеяния туманов и низких слоистых облаков остро стоит для авиации, наземного и морского транспорта, эффективного применения оптико-электронных и тепловизионных систем наведения авиационной техники и т.д. К настоящему времени эта проблема доведена до уровня технологий, которые успешно используются как в России, так и за рубежом, лишь относительно к переохлажденным туманам. Но переохлажденные туманы составляют лишь 5\% от общего количества часов с туманом на земном шаре. В связи с этим наиболее актуальной задачей в борьбе с туманами является разработка методов рассеяния тепльх туманов.

Настоящая работа посвящена искусственному рассеянию теплых туманов и инструментальному определению результатов рассеяния. Нами проведены теоретические исследования и натурные эксперименты по рассеянию туманов с использованием высокотемпературных источников тепла [4]. В качестве таких источников в работе использовались взрывы тепловых зарядов, при которых в тумане возникает облако расширяющихся взрывных газов, которое на некотором расстоянии $\mathrm{R}$ от заряда приобретает в целом правильную шарообразную форму. Эти расширяющиеся газы начинают вихреобразно смешиваться с окружающим туманом за счет турбулентной диффузии и механизма неустойчивости Рихтмайера-Мишкова.

Важной задачей в проблеме активных воздействий (AB) на туманы и низкие слоистые облака с целью их рассеяния является оперативное определение горизонтальных и вертикальных протяженностей просветленной зоны в результате $\mathrm{AB}$. Для решения это задачи нами разработан и испытан действующий макет лазерно-локационного устройства (ЛЛУ) [5]. Это устройство используется в стационарных(наземных) условиях и на борту летательного аппарата (самолета-лаборатории Як-40). Наземные измерения проводились на полигоне ФГБУ «ВГИ» «Кызбурун», где одновременно измерялись дальность видимости в тумане стандартными штатными метеорологическими приборами-фотометрами импульсными ФИ-1. Сравнение результатов измерений показало высокую точность ЛЛУ ( \pm 3 м). Вертикальная протяженность просветленной зоны в туманах и низких слоистых облаках измерялась с борта самолета-лаборатории Як-40 на высотах до $1 \div 2$ км.

\section{Материалы, методы исследований и обсуждения результатов}

1. Искусственное рассеяние теплых туманов с помощыю взрывов тепловых зарядов

Если взрывчатое вещество представлено в виде шара, то радиус этого шара для тротила (плотность $\rho=1660$ кг/м³) и многих других взрывчатых веществ средней мощности можно принять равным [1]. 
$R_{0}=0.05 \sqrt[3]{q}$

$\begin{array}{lll}\text { где } & R_{0}- & \text { выражено в } M, \text { a } \\ & \mathrm{q}- & \text { масса заряда в } к 2 .\end{array}$

Первоначальный радиус $R_{H}$ расширяющихся взрывных газов связан с $R_{0}$ формулой

$$
R_{H}=(11 \ldots 12) R_{0}
$$

Действие взрывных газов проявляется в основном на расстояниях, не превышающих $R_{H}$.

Повышения давления и температуры вблизи центра взрыва настолько значительны, что при взрыве в облаке облачные капли, пересекающие фронт ударной волны, дробятся на более мелкие капельки и испаряются. Перепад давления $\Delta P$ в ударной волне, при котором разрушается капля радиусом $\mathrm{r}$, приблизительно можно оценить на основании формулы Лапласа

$$
\Delta \mathrm{P}=\frac{2 \gamma}{\mathrm{r}}
$$

где $\gamma-$

поверхностное натяжение воды.

Таким образом, можно считать, что облачные капли, радиусы которых превосходят величину $2 \gamma / \Delta P$, будут дробиться в ударной волне с амплитудой $\triangle P$.

Возможность полного испарения облачных капель в ударной волне определяется двумя факторами - величиной избыточной температуры $\Delta T$ и длительностью сохранения состояния повышенного давления в ударной волне.

Зависимость избыточного давления $\Delta P$ в ударной волне от расстояния $R$ до центра взрыва определяется формулой М.А. Садовского [6]

$$
\Delta P=7 \frac{q}{R^{3}}\left(\frac{V}{V_{T}}\right)+2.7 \frac{q^{2 / 3}}{R^{2}}\left(\frac{V}{V_{T}}\right)^{2 / 3}+0.84 \frac{q^{1 / 3}}{R}\left(\frac{V}{V_{T}}\right)^{1 / 3}
$$

где $\quad V_{-} \quad$ удельная энергия взрывчатого веществ;

$V_{T}-\quad$ удельная энергия тротила; 
$R$ выражено в $M$ :

$q-$

в $k 2 ; \triangle P-$ в $а{ }^{\prime} M$.

Подставляя вместо $q$ значение $R_{0}$, определяемое формулой $(1)$, можно записать

$$
\Delta \mathrm{P}=5600\left(\frac{R_{0}}{R}\right)^{3}\left(\frac{V}{V_{T}}\right)+1080\left(\frac{R_{0}}{R}\right)^{2}\left(\frac{V}{V_{T}}\right)^{2 / 3}+16.8 \frac{R_{0}}{R}\left(\frac{V}{V_{T}}\right)^{1 / 3}
$$

Формулы (2) и (3) справедливы также и для поздней стадии распространения ударных волн.

Если взрыв происходит на поверхности грунта, то волна распространяется не в полной сфере, а только в полусфере, и объем воздуха, охватываемый ударной волной, уменьшается вдвое. При этом усиление $\Delta P$ такое, которое соответствует удвоснию величины заряда. Таким образом, при взрыве заряда на поверхности грунта в формуле (2) следует заменить $q$ на $2 q$. В результате получаем:

$$
\Delta \mathrm{P}=14 \frac{q}{R^{3}}\left(\frac{V}{V_{T}}\right)+4.3 \frac{q^{2 / 3}}{R^{2}}\left(\frac{V}{V_{T}}\right)^{2 / 3}+1.1 \frac{q^{1 / 3}}{R}\left(\frac{V}{V_{T}}\right)^{1 / 3}
$$

При удалении от центра взрыва амплитуда ударной волны быстро уменьшается, ударная волна становится слабой. На этой стадии зависимость между $\Delta P$ и приращением $\Delta T$ выражается формулой [7]

$$
\Delta T=\frac{\Delta P}{\rho_{0} C_{p}}
$$

$\begin{array}{lll}\text { где } & \rho_{0}- & \text { плотность невозмущенного воздуха, } \\ & C_{p}- & \text { теплоемкость воздуха при постоянном давлении. }\end{array}$

В таблице 1 представлены значения $\Delta P$ и $\Delta T$ на различных расстояниях $R$ от центра взрыва и при различных значениях веса заряда $q$, при $\rho_{0}=1 \Gamma^{*} \mathbf{M}^{-3}, C_{p}=1$ дж к $\Gamma^{-1}$ град ${ }^{-1}$ и $V=V_{T}$. Нижняя строка в каждой колонке относится к взрыву на поверхности грунта.

Из данных таблицы видно, что при мощных взрывах на расстояниях порядка $150 \ldots 200$ метров от места взрыва температура на фронте ударной волны еще заметно отличается от температуры невозмущенного воздуха. 
Таблица 1.

ЗНАЧЕНИЯ $\triangle \mathrm{P}$ И $\triangle \mathrm{T} \mathrm{ПРИ} \mathrm{РАЗЛИЧНЫХ} \mathrm{R} \mathrm{и} \mathrm{q}$

Table 1. $\triangle P$ and $\triangle T$ values at different $R$ and $q$

\begin{tabular}{|c|c|c|c|c|c|}
\hline \multicolumn{2}{|l|}{$\overline{R, M}$} & 50 & 100 & 150 & 200 \\
\hline \multirow[t]{4}{*}{$q=10 к г$} & $\Delta \mathrm{P}$, атм & 0,042 & 0,020 & 0,012 & 0,009 \\
\hline & & 0,057 & 0,026 & 0,017 & 0,012 \\
\hline & $\overline{\Delta \mathrm{T},{ }^{\circ} \mathrm{C}}$ & 4,20 & 2,0 & 1,2 & 0,9 \\
\hline & & 5,70 & 2,6 & 1,7 & 1,2 \\
\hline \multirow[t]{4}{*}{$\bar{q}=50 \mathrm{kr}$} & $\Delta \mathrm{P}$, атм. & 0,078 & 0,040 & 0,021 & 0,018 \\
\hline & & 0,110 & 0,047 & 0,027 & 0,020 \\
\hline & $\overline{\Delta \mathrm{T},{ }^{\circ} \mathrm{C}}$ & 7,8 & 4,0 & 2,1 & 1,8 \\
\hline & & 11,0 & 4,7 & 2,7 & 2,0 \\
\hline \multirow[t]{4}{*}{$q=100$ кг } & $\Delta \mathrm{P}$, атм. & 0,107 & 0,046 & 0,030 & 0,020 \\
\hline & & $\overline{0,150}$ & 0,062 & 0,038 & 0,034 \\
\hline & $\overline{\Delta \mathrm{T},{ }^{\circ} \mathrm{C}}$ & 10,7 & 4,6 & 3,0 & 2,0 \\
\hline & & 15,0 & 6,2 & 3,8 & 3,4 \\
\hline
\end{tabular}

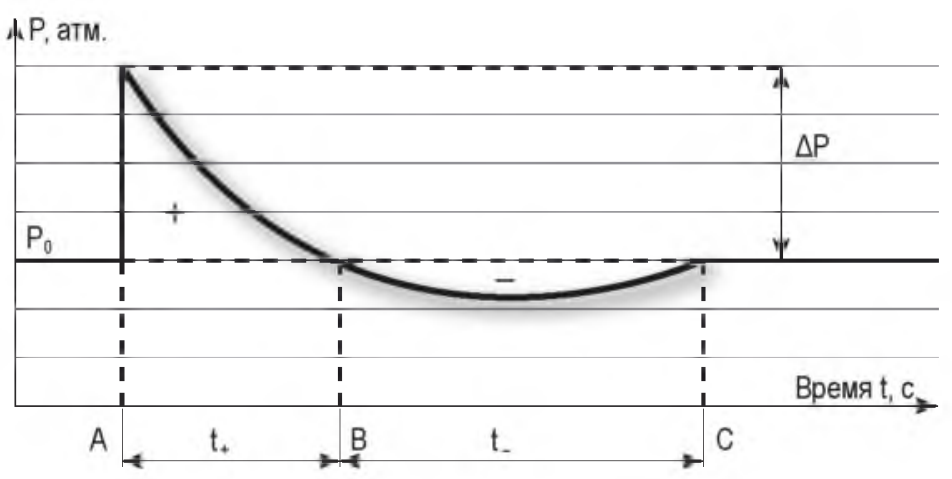

Рис. 1.

Зависимость давления $\mathrm{P}$ от времени $\mathrm{t}$ на фронте сравнительно слабой ударной волны.

$\mathrm{P}_{0}$ - давление в невозмущенном воздухе,

$\Delta \mathrm{P}$ - перепад давления в ударной волне,

$\mathrm{AB}$ - область сжатия ( $\left.\mathrm{t}_{+}\right)$, BC - область разрежения (t.).

Fig. 1. The dependence of the pressure $P$ on the time $t$ at the front of a relatively weak shock wave.

$\mathrm{P}_{0}$ - pressure in the undisturbed air:

$\triangle \mathrm{P}$ is the differential pressure in a shock wave

$A B$ is the area of compression $\left(t_{+}\right)$,

$\mathrm{BC}$ - region of rarefaction $(\mathrm{t})$ 
Если проследить за изменением во времени давления $P$ на определенном расстоянии от центра взрыва, то получается картина, изображенная на рис. 1

Падение давления $\Delta P$ происходит практически по прямолинейному графику. Длившаяся некоторое время зона сжатия (отрезок АВ) сменяется более продолжительной зоной разряжения (отрезок ВC).

Воздух, через который проходит ударная волна, движется в зоне сжатия в ту же сторону, что и волна, а в зоне подсоса - в противоположную сторону.

Скорость движения воздуха непосредственно за фронтом ударной волны равна

$$
v_{\phi}=\frac{\Delta P}{Д_{y} \rho_{0}}
$$

где $\not_{y}-$

скорость ударной волны, определяемая формулой [6]

$$
Д_{y}=C \sqrt{1+0.83 \frac{\Delta P}{P_{0}}},
$$

где $\quad C-\quad$ скорость звука в воздухе:

$P_{0}-\quad$ давление в невозмущенном воздухе.

При $\Delta P \approx 0,04$ атм. из формул (6) и (7) получаем:

$Д_{y} \approx 336,4 M c^{-1}, v_{\phi} \approx 10 M \mathcal{C}^{-1}$ (при $C=331 M \mathcal{C}^{-1}$ ).

Таким образом, скорость массового потока за фронтом слабой ударной волны составляет величину порядка $10 \mathrm{Mc}^{-1}$.

Облачные капли, пересекающие фронт ударной волны, захватываются и увлекаются спутным потоком воздуха, возникающим за ударной волной. В силу малости числа Стокса можно считать, что движение этих капель происходит под действием стоксовой силы $\vec{F}_{C T}=6 \pi \eta r \vec{v}_{r}$, где $\eta-$ динамический коэффициент вязкости воздуха; $v_{r}-$ скорость капель относительно потока воздуха: $v_{r}=v_{\phi}-v$, где $v$ - абсолютная скорость движения облачной капли.

Уравнение движения капли можно записать в виде

$$
m \frac{d v}{d t}=6 \pi \eta r\left(v_{\phi}-v\right),
$$

где $\quad m-$

масса капли. Из этого уравнения получаем:

$$
\frac{d v}{d t}=\frac{9 \rho v}{\rho_{k} r^{2}}\left(v_{\phi}-v\right)
$$

$$
\begin{array}{lll}
\text { где } & v- & \text { кинематическая вязкость воздуха: } \\
& \rho_{k}- & \text { плотность воды. }
\end{array}
$$

Решение уравнения (8), удовлетворяющее начальному условию $v(0)=0$, имеет вид 
$v=v_{\phi}\left(1-e^{-i t}\right)$

где $\tau=\frac{\rho_{k} r^{2}}{9 v \rho} . \quad$ Отсюда при $r=10$ мкм получаем $\tau=5.6 \cdot 10^{-4} \mathrm{c}$, а при $r=1$ мкм $\tau=5.6 \cdot 10^{-6} \mathrm{c}$.

С другой стороны, на основе опытов и теоретических расчетов установлено, что в слабой ударной волне время действия положительного давления в ударной волне $\mathrm{t}_{+}$(участок $\mathrm{AB}$ на рис. 1) связано с весом заряда $q$ и с расстоянием $R$ формулой [6]

$$
t_{+}=\alpha \sqrt{R} \cdot \sqrt[6]{q},
$$

где $\alpha=0.0015\left(\frac{v}{v_{T}}\right)^{1 / 6} ; \quad$ R выражено в м;

$$
\begin{aligned}
& q-\text { в кг; } \\
& \text { t - в сек. }
\end{aligned}
$$

При $R=50 \mathrm{M}, q=10$ кг и $v=v_{T}$ получаем: $t_{+} \approx 1.56 \cdot 10^{-2} c$. В этом случае в формуле (9) отношение $\mathrm{t} / \tau \ll 1$ и $v \cong v_{\phi}$

Из этих оценок следует, что облачные капли, попадающие в спутный поток воздуха за фронтом ударной волны, практически мгновенно увлекаются этим потоком как в зоне сжатия, так и в зоне разрежения. Поэтому процессами взаимной коагуляции этих капель вследствие различия скоростей их движения практически можно пренебречь.

Более существенным является процесс испарения облачных капель за фронтом ударной волны. Если в зоне сжатия газа температуру принять в среднем постоянной, то формулу, описывающую закон изменения радиуса капли, можно записать в виде

$$
r^{2}=r_{0}^{2}-\frac{2 \not \Delta \rho_{n}}{\rho_{k}} t
$$

где $\quad r_{0}-\quad$ начальный радиус капли;

Д- коэффициент диффузии водяного пара в воздухе;

$\rho_{k}-\quad$ плотность воды;

$\Delta \rho_{n}-\quad$ разность плотностей пара на поверхности капли и вдали от нее.

Исходя из уравнения Клапейрона-Клаузиуса можно записать

$$
\Delta \rho_{n} \cong \frac{\rho_{n}\left(T_{0}\right) L \Delta T}{R_{n} T_{0}^{2}}=\frac{\rho_{n}\left(T_{0}\right) L \Delta P}{\rho_{0} C_{P} R_{n} T_{0}^{2}} .
$$

Здесь $T_{0}-\quad$ абсолютная температура воздуха в невозмущенном воздуxe; 
$\rho_{n}\left(T_{n}\right)-\quad$ насыщающая плотность пара при температуре $T_{\theta}$;

$L$ - $\quad$ удельная теплота испарения воды;

$R_{n}-\quad$ газовая постоянная водяного пара;

$\Delta P-\quad$ определяется формулой (4).

Расчеты показывают, что на расстояниях $R$ порядка 30 м и выше основной вклад в формулу (2) вносит последнее слагаемое, т. е.

$$
\Delta P=\frac{\beta q^{1 / 3}}{R}
$$

где $\beta=0.84\left(\frac{v}{v_{T}}\right)^{\frac{1}{3}}$. При этом формула (11) принимает вид

$$
r^{2}=r_{0}^{2}-\frac{2 Д \rho_{n}\left(T_{0}\right) \beta L q^{1 / 3}}{\rho_{k} \rho_{0} C_{P} R_{n} T_{0}^{2} R} t
$$

Полагая в формуле (12) $r=0$, найдем время $\tau$ полного испарения капли

$$
\tau=\frac{\rho_{k} \rho_{0} C_{P} R_{n} T_{0}^{2} r_{0}^{2} R}{2 \beta \rho_{n}\left(T_{0}\right) L q^{1 / 3} \not}
$$

Полное испарение капель имеет место в том случае, когда время $t_{+}$, в течение которого в ударной волне сохраняется перегрев воздуха, больше чем время $\tau$. Таким образом, условие полного испарения капли можно записать в виде

$$
\frac{\rho_{k} \rho_{0} C_{P} R_{n} T_{0}^{2} r_{0}^{2} R}{2 \beta \rho_{n}\left(T_{0}\right) L q^{1 / 3} \not} \leq \alpha \sqrt{R} \cdot \sqrt[6]{q}
$$

Отсюда находим радиус шара $R_{1}$ в облачной среде на высоте $h=30 \ldots 200$ м, внутри которого все капли с радиусами $r \leq r_{0}$ полностью испаряются:

$$
R_{1}=\left(\frac{2 \rho_{n}\left(T_{0}\right) L Д}{\rho_{k} R_{n} T_{0}^{2} \rho_{0} C_{P} r_{0}}\right)^{2}(\alpha \beta)^{2} q
$$

Если же взрыв заряда происходит на поверхности грунта, то в формуле (14) нужно заменить $q$ на $2 q, \beta$ на $\sqrt[3]{2} \beta$ и $\alpha$ на $\alpha \sqrt[6]{2}$. При этом вместо выражения $(\alpha \beta)^{2}$ получаем $2(\alpha \beta)^{2}$ и в формуле (14) появляется дополнительный множитель 4 , т.е. мы имеем

$$
R_{2}=4 R_{1}
$$


Большой интерес представляет также случай, когда заряд взрывается на некоторой высоте $h$ порядка нескольких метров от поверхности земли. В этом случае по облачной среде будут распространяться одна за другой две ударные волны - прямая и отраженная, разделенные промежутком времени $\Delta t=2 h / д_{i}$. При этом для слабых ударных волн избыточное давление на фронте отраженной ударной волны примерно в два раза превосходит избыточное давление в прямой ударной волне. При этом коэффициент $\alpha$ увеличивается в 2 раза. Кроме того промежутки времени $t_{1+}$ и $t_{2+}$, в течение которых сохраняются избыточныс давления в прямой и отраженной ударных волнах, складываются, т. е. коэффициент $\alpha$ в формуле (14) примерно удваивается.

Таким образом, в рассматриваемом случае в формуле (14) появляется дополнительный множитель 16 , т. е. $\mathrm{R}_{3}=16 \mathrm{R}_{1}$.

Запишем теперь характерные значения параметров, входящих в формулу (14):

$T_{0}=280 \mathrm{~K} ; \rho_{0}=1,2 \mathrm{\kappa} \Gamma \cdot \mathrm{M}^{-3} ; \rho_{0}\left(T_{0}\right)=7,8 \cdot 10^{-3} \mathrm{\kappa}^{\cdot} \mathrm{M}^{-3} ; \not=2 \cdot 10^{-5} \mathrm{M}^{2} \mathrm{c}^{-1} ; C_{p}=$

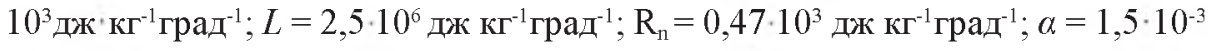
$\mathrm{c}^{\cdot} \mathbf{M}^{-1 / 2} \kappa^{-1 / 6} ; \beta=0,84 \cdot 10^{4} \mathrm{H}^{\cdot} \mathbf{м}^{\cdot} \kappa^{-1 / 3} ; r_{0}=10^{-6} \overline{r_{0}}$, где $\overline{r_{0}}-$ средний радиус облачных капель в мкм. При этих значениях параметров формула (14) принимает вид:

$$
R_{1}=\frac{5}{\bar{r}_{0}^{4}} q\left(\frac{v}{v_{T}}\right)
$$

где $R_{1} \quad$ выражено в м,

$q-\quad$ в кг,

$\overline{r_{0}}-\quad$ в мКм.

При $q=10$ кг, $v=v_{T}$ и $\overline{r_{0}}=1$ мкм по формуле (15) получаем: $R_{1}=50 \mathrm{M}, R_{2}=200 \mathrm{M}, R_{3}=800 \mathrm{M}$.

Эти оценки показывают, что если взрыв газа происходит на поверхности грунта, то полное испарение капель тумана наблюдается в радиусе $R_{2}=200$ м, а на небольшой высоте (1 ..2 м) над грунтом $-R_{3}=800$ м. Минимальный радиус испарения капель тумана $R_{1}=50$ м имеет место на сравнительно больших высотах над уровнем грунта $(30 \ldots 200 \mathrm{M})$.

\section{2. Оценка физической эффективности} активных воздействий на туманы с помощью лазерно-локационного устройства

Проблема оценки результатов активных воздействий на облака и туманы связана с созданием измерительного прибора, позволяющего с высокой точностью измерять геометрические параметры просветленной области в туманах и облаках. Для решения этой задачи нами создан и испытан лазерно-локационный комплекс, включающий в себя следующие устройства $[1,5]$ : 
Оптико-механический тракт и блок накачки, состоящий из лазерного излучателя ИЗ-25-1 с удвоителем частоты излучения и датчиком синхроимпульса, приспособленным для юстировки макета и блока охлаждения излучателя: двух фотоэлектронных умножителей: двух телескопов, снабженных системами поляризационных, абсорбционных (цветных и нейтральных) фильтров; монтажного поворотного стола; юстировочного стола.

2.

Входной тракт, состоящий из логарифмического усилителя (ЛУ), устройства выборки и хранения аналогового сигнала (УВХ) и усилителя мощности (УМ).

3. Двухканальный регистратор аналоговых сигналов (ДРАС). Сигналы с выходов приемного блока поступают на входы двухканального регистратора аналоговых сигналов, каждый канал которого состоит из аналого-цифрового преобразователя (АЦП), оперативного запоминающего устройства (ОЗУ) и схем управления работой.

Лазерно-локационное устройство может быть использовано в наземных условиях и на борту летательных аппаратов.

Определение результатов активных воздействий основано на анализе результатов взаимодействия оптического излучения с рассеивающей аэрозольной (облачной) средой. Лазерно-локационная установка позволяет осуществлять посылку световых импульсов лазерного излучения длительностью до 20 наносекунд и прием обратно рассеянного излучения. При помощи приемной аппаратуры производится регистрация так называемого упругого рассеяния светового излучения, т. е. регистрируется свет той же длины волны, что и зондируюшего светового излучения. Выбор именно упругого рассеяния для анализа результатов АВ обусловлен тем, что интенсивность упругого рассеяния в аэрозольной среде на несколько порядков превосходит интенсивность других типов рассеяния. Это обстоятельство позволяет при ограниченной мощности используемого лазера получить максимально возможную дальность зондирования.

Для определения оптических свойств зондируемого объема был использован анализ формы эхо-сигналов обратного рассеяния, что позволяет проводить сравнительно простые относительные измерения сигнала обратного рассеяния и отказаться от сложной и дорогостоящей калибровки локатора. При анализе результатов зондирования облаков и туманов во внимание не принималось молекулярное рассеяние, так как его вклад на несколько порядков меньше вклада аэрозольного рассеяния. 
Следует выделить два принципиально различных варианта зондирования атмосферы:

1)

2) локатор находится непосредственно в облаках и туманах, подвергаемых АВ;

излучатель находится вне оптически плотного атмосферного образования, например, при пролете самолета с лидаром над слоем облаков, подвергаемых АВ.

Эти два случая резко различаются по подходам к анализу форм эхо-сигналов. В первом варианте световой зондирующий импульс практически полностью рассеивается на расстоянии всего в несколько десятков метров. Действительно, по закону Бугера, мощность оптического излучения $\Phi$, прошедшего сквозь слой рассеивающей среды толщиной $l, \mathrm{c}$ коэффициентом ослабления оптического излучения $\varepsilon$, равна [8]:

$$
\Phi=\Phi_{0} \cdot \mathrm{e}^{e l}
$$

где $\Phi_{0}-\quad$ мощность входящего излучения.

С учетом того, что при зондировании среды оптическое излучение проходит одни и те же аэрозольные образования дважды - в прямом и обратном направлениях, мощность оптического излучения только за счет ослабления в атмосфере уменьшается в $e^{2 \varepsilon l}$ раз. Например, при $\varepsilon l=3$, $e^{2 \varepsilon l}=403$. Максимальное расстояние, с которого лидар, находящийся в облаке, получает эхо- сигнал, примерно соответствует метеорологической оптической дальности. Для облаков с коэффициентом ослабления $50 \mathrm{kм}^{-1}$, эта дальность составляет 60 м. Следовательно, глубина проникновения зондирующего оптического излучения в оптически плотную среду практически совпадает с дальностью видимости в этой среде. Эхо-сигнал лазерного локатора, находящегося в облаке, имеет вид одиночного импульса, длительностью до 1 микросекунды, причем с уменьшением оптической плотности облачного образования (коэффициента ослабления $\varepsilon$ ) эта длительность возрастает. Таким образом, по изменению длительности одиночного импульса при пролете самолета через исследуемую область, подвергнутую активному воздействию, можно судить о степени просветления соответствующего метеорологического образования.

Совершенно иной тип эхо-сигнала регистрируется лазерным локатором во втором варианте, когда он находится в чистой атмосфере, вне облачного образования. Это происходит при полете самолета с лидаром на борту над или под облаками. В этом случае лидар регистрирует как перво- 
начальный относительно слабый импульс значительной длительности - до нескольких микросекунд, так и ряд импульсов от аэрозольных образований, лежащих на трассе зондирования. Самый последний большой импульс соответствует слою, в котором зондирующее излучение окончательно рассеивается. При активном воздействии на облачный слой его граниша в зоне воздействия смещается, а глубина проникновения лазерного излучения в облаке увеличивается. Изменение этих двух параметров и позволяет судить o результатах $\mathrm{AB}$ на облачные образования в случае нахождения самолета в чистой атмосфере.

При проведении наземных испытаний лазерно-локационное устройство устанавливалось на земле, работа велась по нижней поверхности облака. Определяется расстояние до облаков, динамика нижней гранишы, энергетический потенциал прибора, глубина проникновения зондирующего луча в облако, дальность видимости по трассе и другие параметры, позволяющие оценить работоспособность прибора и правильность заложенных при его реализации принципов.

Бортовые испытания проводятся в двух вариантах:

работа через боковые иллюминаторы; в этом варианте проверялась работоспособность аппаратуры при энергообеспечении от бортовых источников питания, а также замерялось реальное энергопотребление; при полете выше верхней границы облаков и при боковом крене самолета (Як-40) 25-30 проводятся те же работы, что и при наземных испытаниях, но по верхней кромке облаков:

работа через световые люки в днище самолета: в этом случае проверялась работоспособность в штатной ситуации.

Методика наземных испытаний следующая. Аппаратура устанавливается на земле в закрытом помещении, имеющем световое окно, которое обеспечивает достаточный обзор по азимуту и углу места. В качестве контрольных средств используются метеорологические средства оборудования полигона (измерители прозрачности, измерители высоты облаков). Испытания проводятся при различных метеорологических ситуациях - при наличии тумана, при низкой и высокой облачности. Контроль правильности определения дальности видимости в атмосфере ведется при работе по горизонтальным трассам в слабом тумане или дымке (при метеорологической дальности видимости, равной 200-500 м). Проверяется совпадение результатов измерений с помощью разработанной аппаратуры, проводится необходимая коррекция. Затем лазерно-локационное устройство переводится на 
работу по наклонным трассам и вертикально в зенит. Измеряется расстояние до нижней кромки облаков (рис. 2), результаты контролируются штатными средствами.

Методика при работе через боковые иллюминаторы следующая. Аппаратура, предварительно испытанная и откалиброванная в наземных условиях, устанавливается на борт самолета-лаборатории. Ведутся измерения прозрачности атмосферы по трассам, параллельным поверхности земли и перпен-
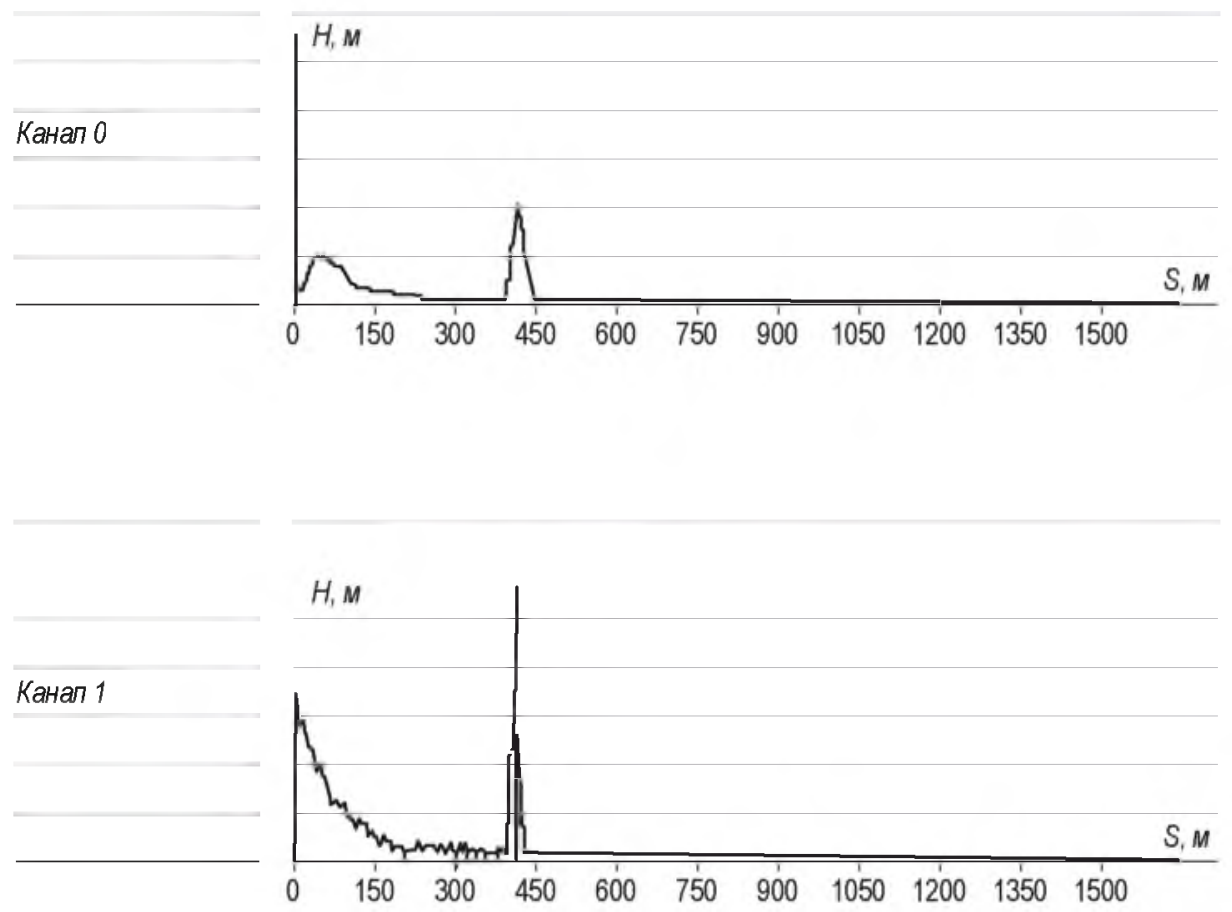

Рис. 2.

Пример записи при определении расстояния до облачных образований H и S. Длина волны $\lambda=0,532$ мкм. В первом канале («Канал 0 )) плоскость пропускания поляроида параллельна плоскости поляризации лазерного излучения. Во втором («Канал 1 ») - перпендикулярна плоскости поляризации.

Fig. 2. An example of a record when determining the distance to cloud formations is $\mathrm{H}$ and S. The wavelength $\lambda=0.532$ microns. In the first channel («Channel $0 »)$ the transmission plane of the polaroid is parallel to the polarization plane of the laser radiation. In the second channel ((Channel 1)) - perpendicular to the polarization plane. 
дикулярным траекториям движения самолета. Необходимо, чтобы самолет двигался в разрывах между облаками, при этом определяется прозрачность атмосферы в разрывах и в облаке, а также дальность до боковых кромок облаков. Ведется проверка работоспособности аппаратуры на борту в режиме длительного использования.

При работе через световые люки в днище самолета-лаборатории аппаратура, отьюстированная в наземных условиях, устанавливается на борт самолета. Зондирование ведется в надир. Метеоситуация - приземный туман. Самолет движется над полигоном на высоте около 1000 м. Во время первых пролетов контролируется работоспособность аппаратуры; в момент пролета над точкой расположения штатной наземной аппаратуры производится сверка результатов; при необходимости производится подстройка. На экране постоянно выводятся в графическом виде результаты дальности видимости по трассам над слоем тумана и в самом слое тумана.

\section{Выводы}

1. Распространение ударных волн в облачной среде приводит к созданию зоны просветления (увеличению метеорологической дальности видимости) вследствие испарения облачных капель под действием повышения температуры и давления на волне сжатия по сравнению с их значениями в невозмущенном воздухе. При мощных взрывах (см. табл. 1) на расстояниях $100 \div 200$ м от места взрыва температура на фронте ударной волны еще существенно отличается от температуры в невозмушенной среде (на 6,2 и $3,2{ }^{\circ} \mathrm{C}$ соответственно). При взрывах заряда $(q=10$ кг) на поверхности земли и на высоте 1 . . 2 м от грунта испарение капель тумана происходит в объеме сферы радиусом до $200 \ldots 800$ м.

2.

Разработан и испытан действующий макет лазерно-локационного устройства (ЛЛУ) с лазерным излучателем ИЗ-251 для контроля и регистрации результатов $\mathrm{AB}$ на туманы и низкие слоистые облака. Работы велись на длине волны излучения $\lambda=0,532$ мкм.

3. Проведены натурные испытания ЛЛУ на борту самолеталаборатории Як-40 и на земле. Результаты этих испытаний сравнивались с результатами измерений, проводившихся одновременно штатными специальными метеорологическими приборами (фотометры импульсные ФИ-1, дистанци- 
онные измерители высоты облаков и др.), установленными на полигоне проведения АВ.

Результаты сравнений показали высокую точность работы ЛЛУ ( $\pm 4 \mathrm{~m})$.

\section{Библиографический cписок}

1. Калов Х.М., Калов Р.Х. К расчету зоны просветления при взрыве тепловых зарядов в облачной среде. Метеорология и гидрология. 2018. №5. С. 18-28.

2. Chernikov A.A. Fog dispersal. Jn: WMP Report No. 31, Technical Document WMO/TD, No. 936. Vol. III. P. 19-32.

3. Weinstein A.J., Kunkel B.A. Fog dispersal. An Operational Weather Modification Technology Today // Papers presented at the 2-nd WMO Scientific Conf. on Weather Modification, Boulder, Colorado. 1976. P. 381-388.

4. Калов Х.М., Калов Р.Х. Физические основы, методы и средства активных воздействий на грозо-градовые облака и туманы: монография. Нальчик: Полиграфсервис и Т, 2010. 220 с.

5. Ашкинадзе Д.А., Белобровик В.И., Сергеев Н.М. Разработка и создание действующего макета устройства контроля и регистрации результатов активных воздействий на туманы и слоистую облачность. Отчет по НИР, 1989. 109 с.

6. Покровский ГИ. Взрыв. М.: Недра, 1967. 411 с.

7. Зельдович Я.Б., Райзер Ю.П. Физика ударных волн и высокотемпературных гидродинамических явлений. М.: Наука, 1966. $687 \mathrm{c}$.

8. Качурин Л.Г. Физические основы воздействия на атмосферные процессы: монография. Ленинград: Гидрометеоиздат, 1978 $455 \mathrm{c}$.

\section{References}

1. Kalov Kh.M., Kalov R.Kh. K raschetu zony prosvetleniya pri vzryve teplovykh zaryadov $\vee$ oblachnoy srede. Meteorologiya i gidrologiya (To the calculation of the zone of enlightenment in the explosion of heat charges in a cloudy environment). 2018. №5. P. 18-28. (in Russ).

2. Chernikov A.A. Fog dispersal. Jn: WMP Report No. 31, Technical Document WMO/TD, No. 936. Vol. III. P. 19-32.

3. Weinstein A.J., Kunkel B.A. Fog dispersal. An Operational Weather Modification Technology Today // Papers presented at the 2-nd WMO Scientific Conf. on Weather Modification, Boulder, Colorado 1976. P. 381-388. 
4. Kalov Kh.M., Kalov R.Kh. Fizicheskiye osnovy, metody i sredstva aktivnykh vozdeystviy na grozo-gradovyye oblaka i tumany: monografiya( Physical bases, methods and means of active influences on thunderstorm-haze clouds and fogs: monograph.). Nal'chik: Poligrafservis i T, 2010. 220 p. (in Russ).

5. Ashkinadze D.A., Belobrovik V.I., Sergeyev N.M. Razrabotka i sozdaniye deystvuyushchego maketa ustroystva kontrolya i registratsii rezul'tatov aktivnykh vozdeystviy na tumany i sloistuyu oblachnost'. (Development and creation of a valid layout of the device for monitoring and recording the results of active impacts on fogs and stratus clouds ) Otchet po NIR, 1989. 109 p. (in Russ).

6. Pokrovskiy G.I. Vzryv (Explosion). Moskva: Nedra, 1967. 411 p. (in Russ).

7. Zel'dovich Ya.B., Rayzer Yu.P. Fizika udarnykh voln i vysokotemperaturnykh gidrodinamicheskikh yavleniy(Physics of shock waves and high-temperature hydrodynamic phenomena). M.: Nauka, 1966. 687 p. (in Russ)

8. Kachurin L.G. Fizicheskiye osnovy vozdeystviya na atmosfernyye protsessy: monografiya (Physical bases of influence on atmospheric processes: monograph).Leningrad: Gidrometeoizdat, 1978. 455 p. (in Russ).

\section{Рукопись поступила в редакцию 23.12.2018 г. Принята к публлкации 25.02.2019 г.}

\section{6 авторах}

Калов Хажбара Мамизович, доктор физико-математических наук, профессор, главный научный сотрудник Федерального государственного бюджетного учреждения «Высокогорный геофизический институт». Адрес: Россия, Кабардино-Балкарская республика, г. Нальчик, пр. Ленина, А. 2, Scopus ID: 6505656548, Researcher ID: V-6492-2018.

Телесон: 8 (928) 080-59-42.

E-mail: ruslan_kalov@mail.ru.

Калов Руслан Хажбарович, кандидат физико-математических наук, доцент, заместитель директора Федерального государственного бюджетного учреждения «Высокогорный геофизический институт» по научной работе. Адрес: Россия, Кабардино-Балкарская республика, г. Нальчик, пр. Ленина, д. 2, Scopus ID: 57202231180, Researcher ID: V-6505-2018.

Телефон: 8 (928) 718-50-14.

E-mail: ruslan_kalov@mail.ru. 


\section{About the authors}

Kalov Khazhbara Mamizovich, Doctor of Physical and Mathematical Science, Professor, chief scientific researcher of the Federal state budgetary institution «High-Mountain Geophysical Institute» Address:

Russia, Kabardino-Balkar Republic, Nalchik, Lenin Ave., 2. Scopus ID: 6505656548.

Researcher ID V-6492-2018.

Phone: 8 (928) 080-59-42

E-mail: ruslan_kalov@mail.ru.

Kalov Ruslan Khazhbarovich, Candidate of Physical and Mathematical Sciences, Associate Professor, Deputy Director for scientific work of the Federal state budgetary institution «High-Mountain Geophysical Institute»

Address:

Russia, Kabardino-Balkar Republic, Nalchik, Lenin Ave., 2

Scopus ID: 57202231180 ,

Researcher ID V-6505-2018,

Phone: 8 (928) 718-50-14

E-mail: ruslan_kalov@mail.ru. 